\title{
A new diatom index to assess ecological quality of running waters: a case study of water bodies in western Anatolia
}

\author{
Assane Anabi Toudjani ${ }^{1}$, Abuzer Çelekli ${ }^{1 *}$, E. Yonca Gümüş ${ }^{1}$, Seda Kayhan ${ }^{1}$, H. Ömer Lekesiz ${ }^{1}$ \\ and Tolga Çetin ${ }^{2}$ \\ 1 Department of Biology, Faculty of Art and Science, University of Gaziantep, 27310 Gaziantep, Turkey \\ 2 T.R. Ministry of Forestry and Water Affairs, Directorate General for Water Management, Ankara, Turkey
}

Received 3 May 2017; Accepted 14 June 2017

\begin{abstract}
Diatoms are an important part of aquatic biodiversity and the main component of phytobenthos. They play a key role in aquatic ecosystems and indicate water quality. The European Union Water Framework Directive requires that phytobenthos be used for the ecological quality assessment of water. This study evaluated the ecological status of various watercourses in the western Mediterranean basin of Turkey using a multivariate approach and presents a new trophic index Turkey (TIT) based on diatom assemblages. Twenty-five running water bodies were seasonally monitored for biological and physicochemical analyses from summer 2014 to summer 2015. A total of 102 species belonging to 22 genera were recorded. Cymbella excisa Kützing, Gomphonella parvula (Kützing) Rabenhorst, Ulnaria ulna (Nitzsch) Compère, and Cocconeis communis f. placentula (Ehrenberg) Chmielevski were the most commonly found species. A canonical correspondence analysis was used to examine the relationship between species and environmental factors. The most effective explanatory factors, including nitrate nitrogen, electrical conductivity, altitude, total nitrogen, orthophosphate, and calcium carbonate significantly influenced the ecological preferences of diatom species in the ecosystems. TIT values ranged between 1.53 in Kaya creek and 2.73 in Dalaman stream (A8). Ecological status of water bodies was assessed using an ecological quality ratio based on trophic index Turkey (EQRTIT) for each of the stations. EQR-TIT ranged from 0.44 in Dalaman stream (A8) to 0.99 in Kocabük creek during the study period. TIT had a high correlation coefficient $\left(R^{2}=0.77\right)$ with log total phosphorus and may be an appropriate diatom metric to assess the ecological status of water bodies.
\end{abstract}

Key words: Phytobenthos / EQR / TIT / Water Framework Directive / Water quality

\section{Introduction}

Water quality is an important parameter in drinking water supply, irrigation, fish production, recreation and other purposes for which the water was impounded (Mustapha, 2008; Wallis et al., 2011). Since the industrial revolution, freshwater quality has deteriorated dramatically due to population growth, industrialization and climate change, leading to severe water pollution and scarcity worldwide (Schindler, 2006; Smol, 2008). Direct sewage inputs, runoff from fertilized soils and land erosion through logging activities have had a major impact on water resources over the last century (Billen et al., 2001; Wunsan et al., 2002; Ducharne et al., 2007; Delgado and Pardo, 2014). The increasing availability of nutrients such as nitrate and phosphate (through fertilizers or sewage) in freshwaters, commonly associated with eutrophication,

*Corresponding author: celekli.a@gmail.com is affecting the productivity and community structure of primary producers in aquatic ecosystems (Leira et al., 2009; Delgado and Pardo, 2014).

In recent decades, significant efforts have been made in many countries to assess water quality using not only physical and chemical parameters but also biological elements (Rott et al., 1999; Padisák et al., 2006; Kelly et al., 2008; Mustapha, 2008). In accordance with the European Union Water Framework Directive (WFD), biological quality elements, including phytoplankton, phytobenthos (especially benthic diatoms), macrophytes, benthic invertebrates, and fish, should be employed as ecological indicators for the assessment of surface waters (Directive, 2000; European Communities, 2009). Among these biological groups, diatoms are widely used in bioassessments due to their short life cycles and rapid response to different stressors (Hering et al., 2006; Bona et al., 2007; Rimet, 2012; Delgado and Pardo, 2014). In this respect, a large number of diatom indices 


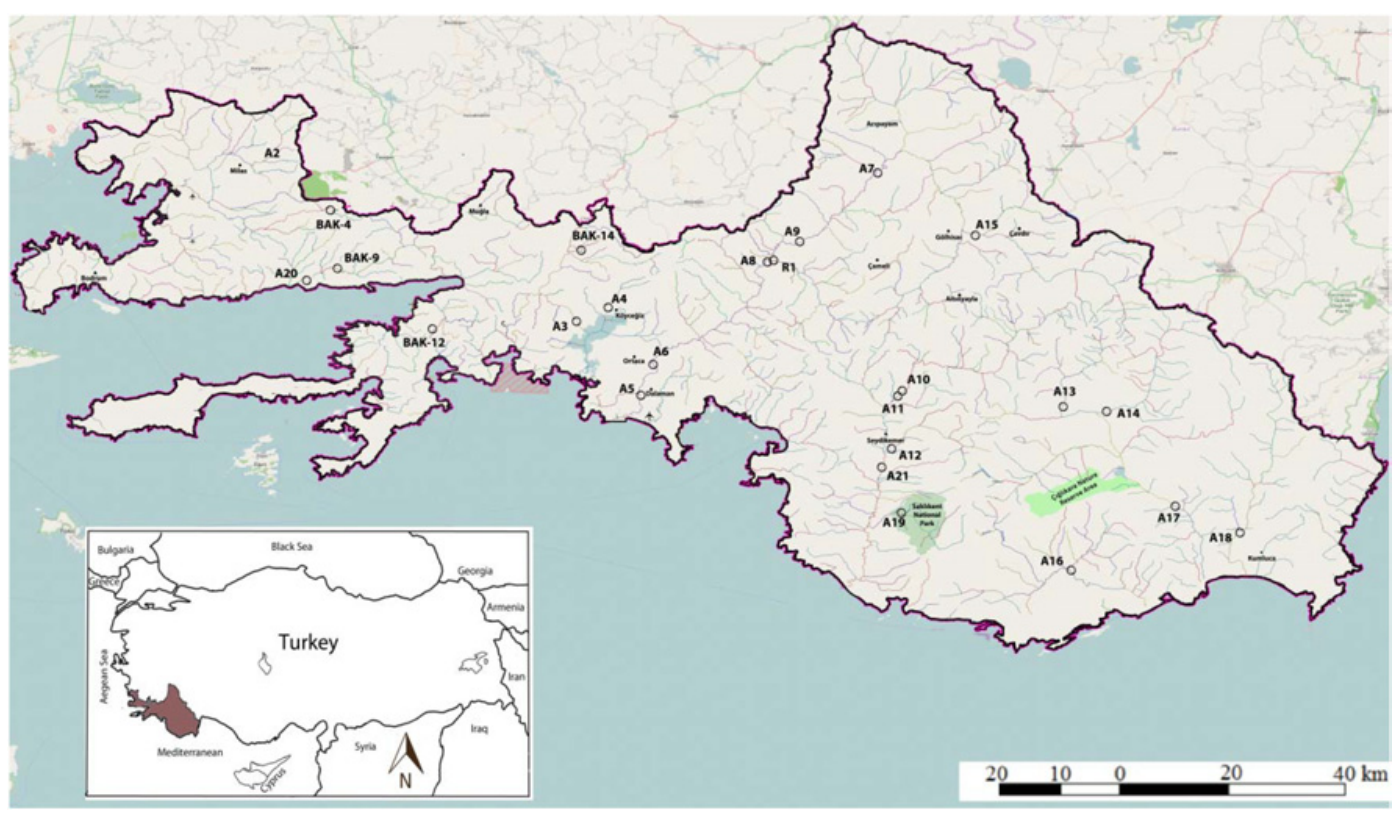

Fig. 1. Location of sampling stations. For station codes: Sarıçay creek, A2; Namnam creek, A3; Dalaman stream, A5-9; Seki stream, A10, A11; Çayiçi creek, A12; Kızılöz creek, A13; Kocadere creek, A14; Çavdır stream, A15; Boğluca stream, A16; Akçay stream, A17; Alakır stream, A18; Eşen stream-1, A19; Kanlı stream, A20; Eşen stream-2, A21; Kaya creek, BAK4; Kocabük creek, BAK9; Karabeyyurdu creek, BAK12; Delin creek, BAK14; R1 creek, R1.

have been developed to estimate water quality in various geographic areas (Potapova et al., 2004).

The studies that used phytobenthos (hereafter related specifically to diatoms) to monitor aquatic ecosystems have mainly investigated running waters (e.g. Round, 1991; Vadeboncoeur and Steinman, 2002; Potapova and Charles, 2003; Rott et al., 2003; Ács et al., 2004; Bellinger et al., 2006; Della Bella et al., 2012; O'Driscoll et al., 2012; Rimet, 2012; Delgado and Pardo, 2014; Leelahakriengkrai and Peerapornpisal, 2014). In this regard, several diatom indices have been developed in different countries to evaluate the ecological status of streams and rivers, including the saprobic index Zelinka and Marvan (1961), indice de polluosensibilité $\approx$ the specific pollution index, SPI (CEMAGREF, 1982), the trophic index, TI (Rott et al., 1999), the Eutrophication and/or Pollution Index Diatom based, EPI-D (Dell'Uomo, 1999, 2004) and the trophic diatom index (TDI) (Kelly and Whitton, 1995; Kelly et al., 2008).

No diatom index for freshwater quality assessment is yet available for Turkish rivers. The direct adoption of foreign diatom index scores can lead to erroneous interpretation of water quality because there are limited overlaps in species composition across different regions (Pan and Stevenson, 1996). The aims of the study were to: (i) define the most important structuring factors of diatom species composition, and (ii) test the suitability and applicability of a recently developed diatom-based metric called the TIT as a potential tool for the ecological status assessment of Mediterranean running water bodies and its performance compared with EPI-D (Dell'Uomo, 1999, 2004), an index developed in another Mediterranean country, Italy.

\section{Materials and methods}

\section{Study area and sampling}

The studied water bodies were located in the western Mediterranean basin of Turkey (Fig. 1). Water and diatom samples were collected seasonally from 25 running water bodies from summer 2014 to summer 2015. Environmental variables such as water temperature, $\mathrm{pH}$, electrical conductivity (EC), and salinity were measured in situ using a YSI professional plus oxygen-temperature meter from just below the surface water. Geographical data (altitude, latitude, and longitude) were recorded using a geographical positioning system (Garmin Vista $\mathrm{HCx}$ model GPS) during the study period. The water flow was measured using a StreamPro ADCP (Acoustic Doppler Current Profiler). The epilithic diatoms were collected according to European standard sampling methods for limnetic systems (Kelly et al., 1998; European Committee for Standardization, 2003, 2004a, b; European Communities, 2009). Epilithic samples were collected in riffle areas with normal hydrological condition (not disturbed) by scraping the upper surface of the stones using a toothbrush. The samples were fixed using Lugol's iodine solution immediately after collection (European Committee for Standardization, 2004a, b).

\section{Laboratory techniques}

Water samples were stored in iceboxes with ice packs and transferred to the laboratory for later analyses. Chemical variables such as total nitrogen (TN), ammonium nitrogen $\left(\mathrm{N}-\mathrm{NH}_{4}\right)$, nitrate nitrogen $\left(\mathrm{N}-\mathrm{NO}_{3}\right)$, 
nitrite nitrogen $\left(\mathrm{N}-\mathrm{NO}_{2}\right)$, total phosphorus (TP), orthophosphate $\left(\mathrm{P}^{-} \mathrm{PO}_{4}\right)$, biological oxygen demand $\left(\mathrm{BOD}_{5}\right)$, and calcium carbonate $\left(\mathrm{CaCO}_{3}\right)$ were measured using the standard methods (APHA, 2012).

Permanent slide mounts were prepared for each sample using the standard methods (Kelly et al., 1998; European Committee for Standardization, 2004a, b; European Communities, 2009) to enumerate diatom species. The algae were later examined at $1000 \times$ magnification to estimate the relative abundance of taxa using a compound microscope (Olympus BX53) equipped with a digital camera (DP73 model) and imaging software (Olympus CellSens Vers. 1.6). Diatoms were identified to species level using taxonomic keys (Krammer and Lange-Bertalot, 1991a, b, 1999a, b; Krammer, 2000, 2002; Lange-Bertalot, 2001). Relative percentages of diatom species were calculated and used for statistical analyses and assessment of water quality. To develop the TIT index (Çelekli et al., 2017), data collected from 225 running water bodies from eight basins of Turkey between 2014 (summer and fall) and 2015 (spring and summer) were used. A total of 226 diatom species were identified and the percentages of each species at each sampling station were to be determined. According to the distribution of diatom species based on TP classes, trophic weight and indicator values were determined as described by Rott et al. (1999) and Binder (2001). The TIT value was calculated using the following equation:

$$
T I T=\frac{\sum_{i=1}^{n} b_{i} \times e_{i} \times c_{i}}{\sum_{i=1}^{n} e_{i} \times c_{i}}
$$

where, $b_{i}: i$-taxon trophic weight based on TP classes (1-5); $e_{i}$ : $i$-taxon indicator value $(0-5)$; and $c_{i}$ : the percentage of valves of $i$-species in a sample.

TIT values ranging from 0 to 4 were then expressed as ecological quality ratios (EQRs) for the ecological quality assessment of running water bodies according to the WFD. Boundary classes were deteremined according to the WFD, using the box-plot analysis for each typology (Çelekli et al., 2017). EQR is given by (Equation (2))

$$
\mathrm{EQR}=\frac{\left(4-\mathrm{TIT}_{\mathrm{o}}\right)}{\left(4-\mathrm{TIT}_{\mathrm{ref}}\right)}
$$

where $\mathrm{TIT}_{\mathrm{o}}$ and $\mathrm{TIT}_{\text {ref }}$ are the observed and reference TIT, respectively. The EQR values ranged from 0 (poor ecological status) to 1 (high ecological status).

TIT was then compared with the Eutrophication and/or Pollution Index - Diatom based (EPI-D) (Dell'Uomo, 1999, 2004) to evaluate its ability to assess water quality.

\section{Multivariate analyses}

Detrended correspondence analysis (DCA), which is a unimodal response before performing CCA (Leps and Smilauer, 2003) was used at a gradient length more than 3.0. A direct gradient analysis technique, canonical correspondence analysis (CCA), was used to examine the relationship between the response variables (diatoms) and predictor variables (environmental factors) in 25 running water bodies (ter Braak and Šmilauer, 2002). Environmental variables were transformed $(\ln (x+1)$, except for $\mathrm{pH}$ ) to decrease skewness (ter Braak and Šmilauer, 2002). A total of eight environmental variables were used for CCA. The significance of environmental variables to clarify the variance of diatom data in CCA was tested using the forward selection of Monte Carlo simulations with 499 unrestricted permutations. Only species weight range with a percentage of over $1 \%$ were selected for the CCA diagram. CCA was performed using the CANOCO program (ter Braak and Šmilauer, 2002).

\section{Results}

\section{Physicochemical variables}

The results for environmental variables at the sites are given in Table 1 . The water bodies had alkaline water with $\mathrm{pH}$ ranging between 8.1 and 8.7. Delin creek had the lowest mean temperature $\left(14.2{ }^{\circ} \mathrm{C}\right)$, whereas Çayiçi creek had the highest $\left(23.8^{\circ} \mathrm{C}\right)$.

With regard to nutrients, Dalaman stream (A8) had the highest mean values for TN $\left(4029.7 \mu \mathrm{g} . \mathrm{L}^{-1}\right)$, $\mathrm{N}-\mathrm{NO}_{3}\left(1503.3 \mu \mathrm{g} . \mathrm{L}^{-1}\right)$, TP $\left(783.3 \mu \mathrm{g} . \mathrm{L}^{-1}\right)$, and P-PO (341.7 $\left.\mu \mathrm{g} . \mathrm{L}^{-1}\right)$, while the lowest values for TN $\left(149.5 \mu\right.$ g. $\left.\mathrm{L}^{-1}\right), \quad \mathrm{N}-\mathrm{NO}_{3} \quad\left(100 \mu \mathrm{g} . \mathrm{L}^{-1}\right)$, and $\mathrm{P}^{-\mathrm{PO}_{4}}$ (10 $\mu \mathrm{g} . \mathrm{L}^{-1}$ ) were measured in Kaya creek (Bak4), followed Kocabük and Kanlı streams.

The Dalaman stream had a permanent and highest water flow (e.g. $36.8 \mathrm{~m}^{3} . \mathrm{s}^{-1}$ at A6 and $34.8 \mathrm{~m}^{3} . \mathrm{s}^{-1}$ at A5). The highest salinity $(0.43 \mathrm{ppt})$ and EC $\left(776.5 \mu \mathrm{S} . \mathrm{cm}^{-1}\right)$ values were also found in Dalaman stream (A8), while the lowest values of $0.10 \mathrm{ppt}$ and $179.4 \mu \mathrm{S} . \mathrm{cm}^{-1}$, respectively, were measured in Sarıçay creek.

\section{Diatom assemblages}

A total of 102 species belonging to 22 genera mostly dominated by Navicula and Cymbella were recorded (Table 2). Some species such as Fragilaria capucina Desmazières, Cymbella excisa Kützing, Gomphonella parvula (Kützing) Rabenhorst, Ulnaria ulna (Nitzsch) Compère, and Cocconeis communis f. placentula (Ehrenberg) Chmielevski were commonly found in the watercourses during the study period. On the other hand, Navicula scutelloides var. disculus (Schumann) Torka, Diploneis modica Hustedt, and Surirella ovata var. pinnata (W.Smith) Hustedt were rarely observed.

\section{Species-environment relationships with multivariate analyses}

The unimodal response of diatom taxa to environmental variables is found with gradient length, which 


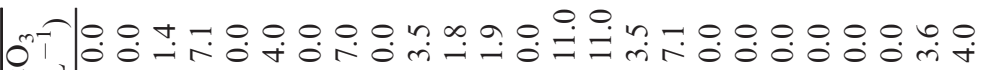
$\int_{0}+1+1+1+1+1+1+1+1+1+1+1+1+1+1+1+1+1+1+1+1+1+1+1+1+1$ U

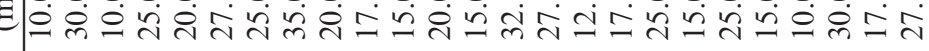

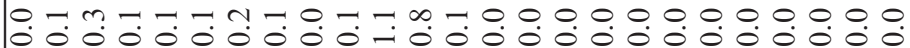
告 $+1+1+1+1+1+1+1+1+1+1+1+1+1+1+1+1+1+1+1+1+1+1+1+1+1$

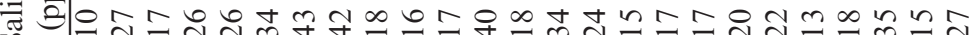

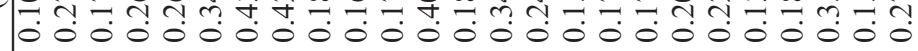

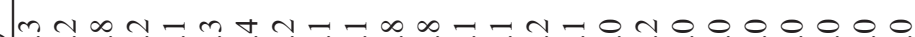

r 0000000000000

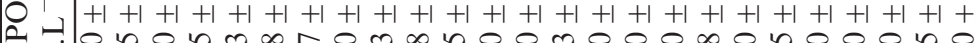
$\therefore$ a

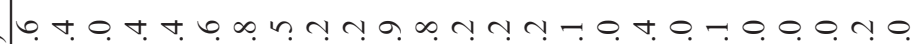

I $00-0000000-m 0000000000000$

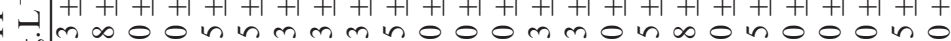

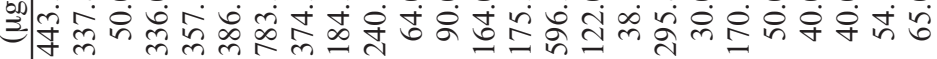

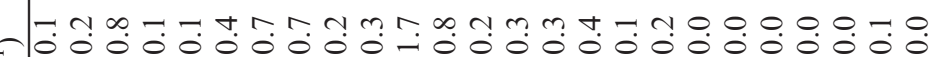
$01+1+1+1+1+1+1+1+1+1+1+1+1+1+1+1+1+1+1+1+1+1+1+1+1+1$ $Z_{0 j}=n$

00 o o o

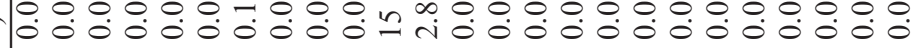

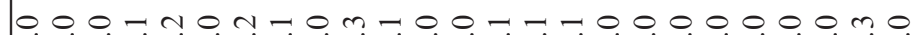

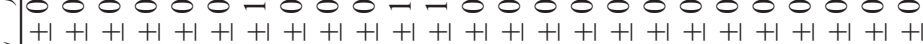

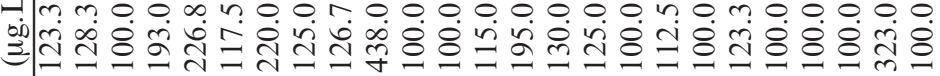

$m m-70 m a m-n-m \infty m 04 m 00000-1$

ح

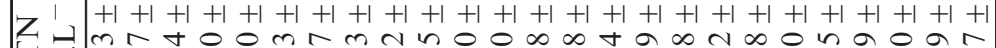

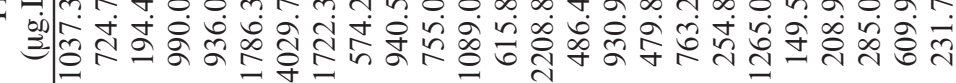

f $0.1+1+1+1+1+1+1+1+1+1+1+1+1+1+1+1+1+1+1+1+1+1+1+1+1+1$

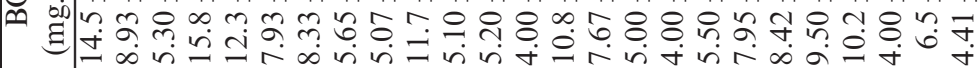

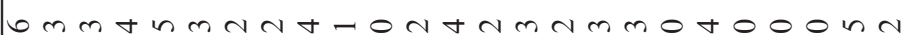

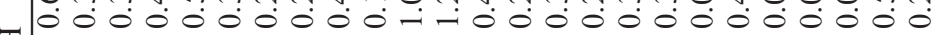
$20+1+1+1+1+1+1+1+1+1+1+1+1+1+1+1+1+1+1+1+1+1+1+1+1+1$

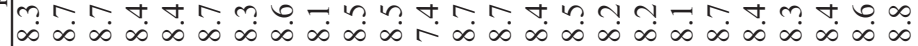
1 0 麦 $+1+1+1+1+1+1+1+1+1+1+1+1+1+1+1+1+1+1+1+1+1+1+1+1+1$

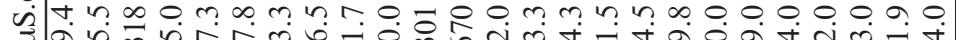

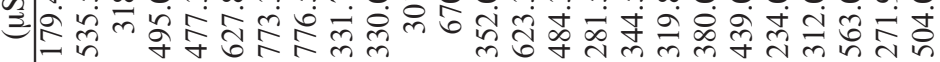

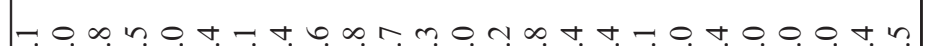

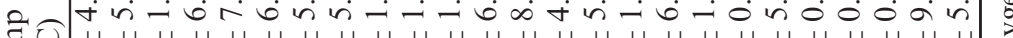

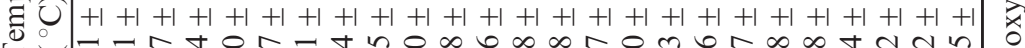

$\infty$ iे

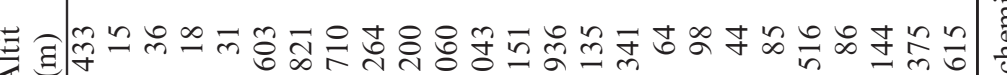


Table 2. List of diatom species and varieties found at the studied sites.

\begin{tabular}{|c|c|c|c|}
\hline Species & Code & Species & Code \\
\hline Achnanthes impexa (Lange-Bertalot) & Acim & Dickieia expecta (VanLandingham) D.G. Mann & Diex \\
\hline Aulocoseira granulata (Ehrenberg) Simonsen & Augr & Diploneis modica (Hustedt) & Dimod \\
\hline Bacillaria obtusa (W.Smith) Elmore & Baob & Encyonema minutum (Hilse) D.G. Mann & Enmi \\
\hline Bacillaria vulgaris (Bory) Ehrenberg & Bavu & Encyonema silesiacum (Bleisch) D.G. Mann & Ensi \\
\hline Clevamphora ovalis (Kützing) Mereschkowsky & Clov & Epithemia adnata (Kützing) Brébisson & Epad \\
\hline Cocconeis communis f. placentula (Ehrenberg) Chmielevski & Coco & Epithemia cistula (Ehrenberg) Ralfs & Epar \\
\hline Cocconeis placentula var. lineata (Ehrenberg) Van Heurck & Coplv & Epithemia frickei (Krammer) & Epfr \\
\hline Cocconeis pumila (Kützing) & Сори & Eunotia exigua var. tenella (Grunow) Nörpel \& Alles & Euex \\
\hline Cocconeis scutellum (Ehrenberg) & Cosc & Fragilaria biceps (Ehrenberg) & Frbi \\
\hline Cocconema helveticum (Kützing) West \& G.S.West & Cohe & Fragilaria capucina (Desmazières) & Frca \\
\hline Craticula accomoda (Hustedt) D.G. Mann & Crac & Fragilaria dilatata (Brébisson) Lange-Bertalot & Frdi \\
\hline Craticula halophila (Grunow) D.G. Mann & Crha & $\begin{array}{l}\text { Gomphonema acuminatum f. laticeps (Ehrenberg) } \\
\text { Ant. Mayer }\end{array}$ & Goac \\
\hline Cyclotella iris (Brun \& Héribaud-Joseph) & Cyir & Gomphonema angustum (C. Agardh) & Goan \\
\hline Cyclotella tibetana $($ Hustedt) & Cyti & Gomphonema gracile (Ehrenberg) & $G o g r$ \\
\hline Cymbopleura amphicephala (Nägeli) Krammer & Cyam & Gomphonema minutum (C. Agardh) C. Agardh & Gomi \\
\hline $\begin{array}{l}\text { Cymbopleura naviculiformis (Auerswald ex Heiberg) } \\
\text { Krammer }\end{array}$ & Cyna & Gomphonella parvula (Kützing) Rabenhorst & Gopa \\
\hline Cymatopleura solea (Brébisson) W. Smith & Cyso & Gomphonema pseudoaugur (Lange-Bertalot) & Gops \\
\hline Cymbella cistula (Hemprich) Kirchner & Cyci & Gomphonema truncatum (Ehrenberg) & Gotr \\
\hline Cymbella cymbiformis (Agardh) & $C y c y$ & $\begin{array}{l}\text { Gomphonema truncatum var. clavatum } \\
\text { (Ehrenberg) Eberle }\end{array}$ & Gotrc \\
\hline Cymbella ehrenbergii (Kützing) & Cyeh & Halamphora subcapitata (Kisselew) Levkov & Hasu \\
\hline Cymbella excisa (Kützing) & Cyex & Hantzschia distinctepunctata var. circuligera (Compère) & Hadi \\
\hline Cymbella hantzschiana (Krammer) & Cyha & Hantzschia weyprechtii (Grunow) & Hawe \\
\hline Cymbella mesiana (Cholnoky) & Cymes & Homoeocladia sigmoidea (Nitzsch) Elmore & Hosi \\
\hline Cymbella simonseni (Krammer) & Cyst & Homoeocladia umbonata (Ehrenberg) Kuntze & Houm \\
\hline Cymbella tumida (Brébisson) van Heurck & Cytu & Lysigonium lineatum (Dillwyn) Trevisan & Lyli \\
\hline Diatoma elongata subsp.tenuis(C. Agardh) Skabichevskii & Diel & Lysigonium varians (C. Agardh) De Toni & Lyva \\
\hline Diatoma hyemalis (Roth) Heiberg & Dihy & Navicula bryophila (J.B. Petersen) & $\mathrm{Nabr}$ \\
\hline Diatoma moniliformis (Kützing) D.M. Williams & Dimo & Navicula capitatoradiata (H. Germain) & $\mathrm{Naca}$ \\
\hline Navicula cincta (Ehrenberg) Ralfs in Pritchard & $\mathrm{Naci}$ & Placoneis clementis (Grunow) E.J. Cox & Plcl \\
\hline Navicula constans (Hustedt) & Nacon & Pleurosigma acuminatum (Kützing) Grunow & Plac \\
\hline Navicula cryptocefalsa (Lange-Bertalo) & $\mathrm{Nacr}$ & Scalptrum attenuatum (Kützing) Kuntze & Scat \\
\hline Navicula cryptocephala f. Veneta (Kützing) Hustedt & Nacrv & Schizonema gregarium (Donkin) Kuntze & Scgr \\
\hline Navicula cryptonella (Lange-Bertalot) & Nacry & Schizonema peregrinum (Ehrenberg) Kuntze & Scpe \\
\hline Navicula digitoradiata (Gregory) Ralfs in Prichard & Nadi & Schizonema phoenicenterum (Ehrenberg) Kuntze & Scph \\
\hline Navicula heimansii (van Dam \& Kooyman) & Nahe & Schizonema radiosu (Kützing) Kuntze & Scra \\
\hline Navicula jaagii (Meister) & Naja & Stephanocyclus meneghinianus (Kützing) Skabitschevsky & Stme \\
\hline Navicula lanceolata (C. Agardh) Kützing & Nala & Surirella brebissonii (Krammer \& Lange-Bertalot) & Subr \\
\hline Navicula margalithii (Lange-Bertalot) & Nama & Surirella biseriata f. Amphioxys (W. Smith) Hustedt & Subi \\
\hline Navicula oppugnata (Hustedt) & Naop & Surirella ovata var. Pinnata (W. Smith) Hustedt & Suop \\
\hline Navicula phylepta (Kützing) & Naph & Surirella ovata var. ovalis (Brébisson) Kirchne & Suoo \\
\hline Navicula radians (Héribaud-Joseph) & Nara & Surirella ovata var. angusta (Kützing) A. Cleve & Suon \\
\hline Navicula recens (Lange-Bertalot) Lange-Bertalot & Nare & Surirella subsalsa (W. Smith) & Susu \\
\hline Navicula schoenfeldii (Hustedt 1930) & Nasc & Ulnaria ulna (Nitzsch) Compère & Ulul \\
\hline Navicula scutelloides var.disculus (Schumann) Torka & $\mathrm{Nascu}$ & & \\
\hline Navicula slesvicensis (Grunow in van Heurck) & Nasl & & \\
\hline Navicula tripunctata (O.F. Müller) Bory & Natr & & \\
\hline Navicula trivialis (Lange-Bertalot) & Natri & & \\
\hline Navicula tuscula (Ehrenberg) & Natus & & \\
\hline Navicula vitabunda (Hustedt in Pascher) & Navi & & \\
\hline Naviculadicta laterostrata (Hustedt) Lange-Bertalot & Nalat & & \\
\hline Nematoplata crotonensis (Kitton) Kuntze & $\mathrm{Necr}$ & & \\
\hline Nitzschia angustata (W.Smith) Grunow & Nian & & \\
\hline Nitzschia brevissima (Grunow in Van Heurck) & Nibr & & \\
\hline Nitzschia elongata (Hassal) & Niel & & \\
\hline Nitzschia palea var. dissipata (Kützing) Schonfeldt & Nipa & & \\
\hline Nitzschia vermicularis var. flexa (Schumann) Cleve-Eule & Nifl & & \\
\hline $\begin{array}{l}\text { Nitzschia vitrea var. recta (Hantzsch; Grunow) } \\
\text { van Heurck }\end{array}$ & Nivi & & \\
\hline Odontidium elegans (Kützing) O’Meara 1875 & Odel & & \\
\hline
\end{tabular}


Table 3. Summary of canonical correspondence analysis (CCA) using a Monte Carlo permutation test for epilithic diatom speciesenvironment variable relationships.

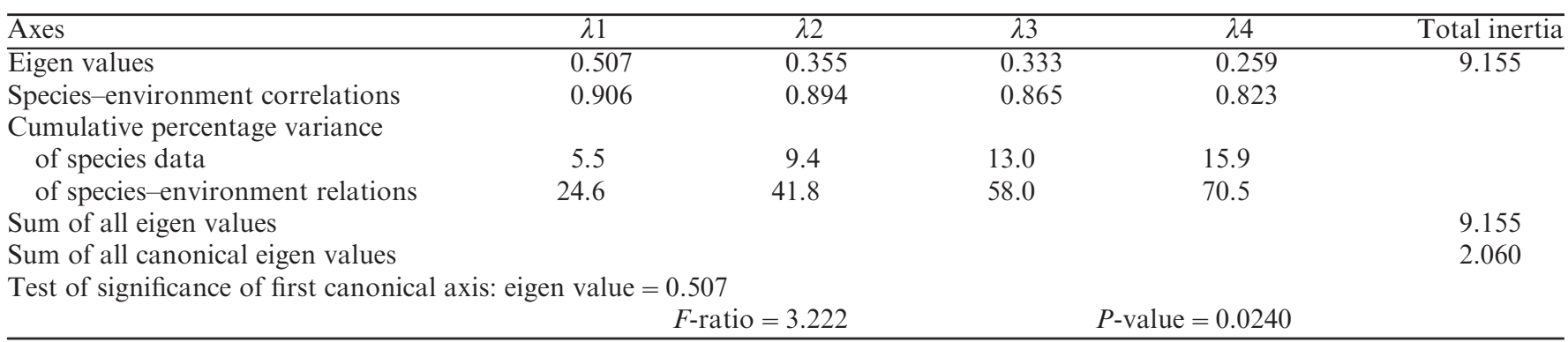

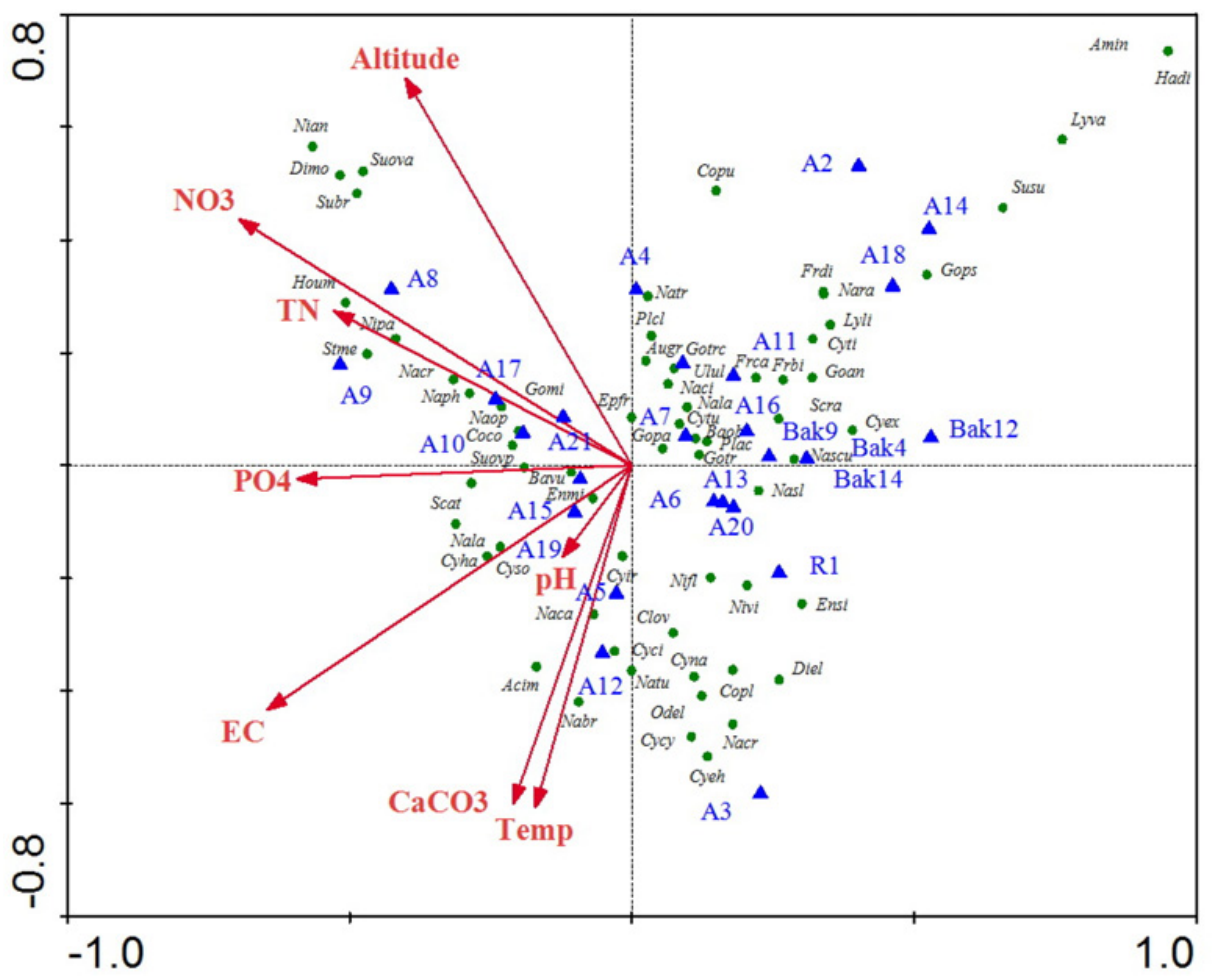

Fig. 2. CCA plot of species-environment relationships at the sampling stations (up triangular). For station codes, see Fig. 1 and for species, see Table 2.

was more than 3.0. After that, CCA analysis was performed to elucidate relationships between diatom species and environmental factors during the study period. $9.4 \%$ of the cumulative variance in the diatom species was explained by the CCA first two axies with $89.4 \%$ the species-environment correlation. Application of the forward selection using the Monte Carlo test confirmed that the first two axes were highly significant $(P=0.024$, $F=3.222$, Table 3 ). The most effective explanatory factors were $\mathrm{N}-\mathrm{NO}_{3}, \mathrm{EC}$, altitude, TN, $\mathrm{P}-\mathrm{PO}_{4}$, and $\mathrm{CaCO}_{3}$ $(P<0.01)$.

Species distribution in relation to physical and chemical variables is given in Figure 2. The ordination of the CCA indicated that predictor variables (environmental factors) affect the distribution of diatom assemblages in the running water bodies of western Anatolia. As shown in Figure 2, nutrient-rich stations such as Dalaman stream
(A8, A9, and A5) and Çavdır stream were distributed in the negative part of the first axis. On the contrary, low nutrient sampling stations (e.g. Kocabük, R1, Kaya, Delin, Namnam, and Karabeyyurdu creeks and Kanl stream) were located in the opposite part. Nitzschia angustata (W.Smith) Grunow, Surirella brebissonii Krammer \& Lange-Bertalot, Diatoma moniliformis (Kützing) D.M.Williams, and others were associated with high altitude and high concentration of nitrogen variables. A few diatoms such as Scalptrum attenuatum (Kützing) Kuntze and $S$. ovata var. pinnata (W.Smith) Hustedt were related to high water $\mathrm{EC}$ and $\mathrm{P}-\mathrm{PO}_{4}$. C. excisa Kützing, Schizonema radiosum (Kützing) Kuntze, N. scutelloides var. disculus (Schumann) Torka and Navicula slesvicensis Grunow in van Heurck were abundant in Kaya, Kocabük, Karabeyyurdu, and Delin creeks, which had relatively low nutrients and EC (Fig. 2). 
Table 4. TIT, EQR, and EPI-D values and ecological status of the stations. Typology codes (A, flow; R, altitude; J, geology; E, slope; Y, precipitation and D, drainage).

\begin{tabular}{|c|c|c|c|c|c|c|}
\hline Stations & Code & Typology & TIT & EQR & Status & EPI-D \\
\hline Sarıçay creek & $\mathrm{A} 2$ & A1R1E2Y2D1J2 & 2.18 & 0.64 & Moderate & 1.28 \\
\hline Namnam creek & A3 & A2R1E1Y2D1J2 & 2.34 & 0.76 & Good & 1.19 \\
\hline Kargıcak creek & A4 & A1R2E2Y2D1J2 & 2.11 & 0.61 & Moderate & 1.40 \\
\hline Dalaman stream & A5 & A2R1E1Y2D2J1 & 2.33 & 0.63 & Moderate & 1.29 \\
\hline Dalaman stream & A6 & A2R1E1Y2D2J2 & 2.28 & 0.77 & Good & 1.18 \\
\hline Dalaman stream & A7 & A2R2E2Y2D2J2 & 2.48 & 0.71 & Moderate & 1.39 \\
\hline Dalaman stream & A8 & A2R2E1Y2D2J2 & 2.73 & 0.44 & Poor & 1.37 \\
\hline Dalaman stream & A9 & A2R2E1Y2D2J1 & 2.66 & 0.61 & Moderate & 1.88 \\
\hline Seki stream & A 10 & A2R2E2Y2D1J1 & 2.50 & 0.84 & Good & 1.22 \\
\hline Seki stream & A11 & A2R2E1Y2D1J1 & 2.36 & 0.65 & Moderate & 1.25 \\
\hline Çayiçi creek & A12 & A2R3E2Y2D1J1 & 2.21 & 0.8 & Good & 1.29 \\
\hline Kızılöz creek & A13 & A2R3E1Y2D1J1 & 2.13 & 0.64 & Moderate & 1.19 \\
\hline Kızılöz creek & A14 & A1R2E1Y2D1J1 & 2.28 & 0.69 & Moderate & 1.15 \\
\hline Çavdır stream & A 15 & A2R2E1Y2D1J2 & 2.62 & 0.61 & Moderate & 1.35 \\
\hline Boğluca stream & A16 & A2R1E1Y2D1J1 & 2.56 & 0.83 & Good & 1.33 \\
\hline Akçay stream & A17 & A2R3E2Y2D2J1 & 2.40 & 0.80 & Good & 1.55 \\
\hline Alakir stream & A18 & A1R1E1Y2D1J1 & 2.13 & 0.83 & Good & 1.36 \\
\hline Eşen stream & A19 & A2R2E2Y2D1J2 & 2.30 & 0.78 & Good & 1.10 \\
\hline Kanlı stream & A20 & A1R1E1Y2D1J1 & 1.85 & 0.93 & High & 1.32 \\
\hline Eşen stream & A21 & A2R1E1Y2D2J2 & 2.36 & 0.77 & Good & 1.50 \\
\hline Karabeyyurdu creek & BAK 12 & $\mathrm{R} 1 \mathrm{D} 2 \mathrm{~A} 1 \mathrm{~J} 2$ & 1.98 & 0.96 & High & 1.07 \\
\hline Delin creek & BAK 14 & A2R1E1Y2D1J2 & 1.88 & 0.98 & High & 1.03 \\
\hline Kaya creek & BAK4 & A2R1E1Y2D1J1 & 1.53 & 0.99 & High & 1.01 \\
\hline Kocabük creek & BAK9 & A1R1E1Y2D1J1 & 1.89 & 0.99 & High & 1.28 \\
\hline R1creek & $\mathrm{R} 1$ & $\mathrm{R} 2 \mathrm{D} 2 \mathrm{~A} 2 \mathrm{~J} 2$ & 1.84 & 0.91 & High & 1.18 \\
\hline
\end{tabular}

\section{Ecological status}

Results of the TIT, and its related EQR and EPI-D values are presented in Table 4 . The TIT values of the water bodies varied from 1.53 in Kaya Creek to 2.73 in Dalaman stream (A8). To compare the diatom indices in the water bodies, TIT and EPI-D were regressed against $\log$ TP. These relationships are shown in Fig. 3(a) and (b), respectively. Based on $R^{2}$ of the regresions, a higher proportion of variance of TIT was explained by $\log$ TP compared with that of EPI-D. Therefore, the TIT was selected for EQR calculation. The ecological status of the studied sites was evaluated using the EQR value based on the TIT. Kocabük creek had the highest EQR-TIT value $(0.99)$, while the lowest value $(0.44)$ was found in Dalaman stream (A8). The ecological status ranged from high to poor. Poor ecological status was observed only in Dalaman stream (A8) (Table 4), whereas high ecological status was found in Kanlı stream, Kocabük, R1, Kaya, Delin, and Karabeyyurdu creeks. Moreover, the EQR values based on the TIT also showed a negative significant correlation with $\operatorname{logTP}\left(R^{2}=0.67, P<0.01\right)$ (Fig. 3(c)).

\section{Discussion}

\section{Physicochemical variables}

During the present study, Dalaman stream (A8) had higher nutrient concentrations compared to the other watercourses. This station was under pressure from human activities in Acıpayam District and several villages, and from farming processes. We suggested that the water quality of Dalaman stream could be deteriorated due to excessive addition of inorganic and organic compounds from municipial waste discharges, agricultural practices, livestock, and polluted runoff. It has already been shown that anthropogenic activities affect diatom composition and water quality (e.g., Van Dam et al., 1994; Reynolds et al., 2002; Ács et al., 2004; Bellinger et al., 2006; Hlúbiková et al., 2007; Padisák et al., 2009; Delgado and Pardo, 2014). High population densities and the multiplicity of industrial and agricultural activities expose most hydrographic basins close to large urban centers to heavy and rising environmental impacts, especially to pollution by domestic and industrial waste residues (Mancini and Arcà, 2000; Salomoni et al., 2006). In contrast, the lowest nutrient concentrations were measured in Kaya, Kocabük, R1, and Karabeyyurdu creeks. These ecosystems represented undisturbed conditions and their water beds were mainly composed of stones, gravel, and sand.

\section{Structuring factors of diatom community composition}

A total of 102 taxa mostly belonging to Navicula and Cymbella were identified in the studied area during the study period. The diatom taxa identified in this study had mostly been reported previously in other running water bodies in the USA, Italy, Tanzania, and Ireland (Potapova and Charles, 2003; Bellinger et al., 2006; Della Bella et al., 


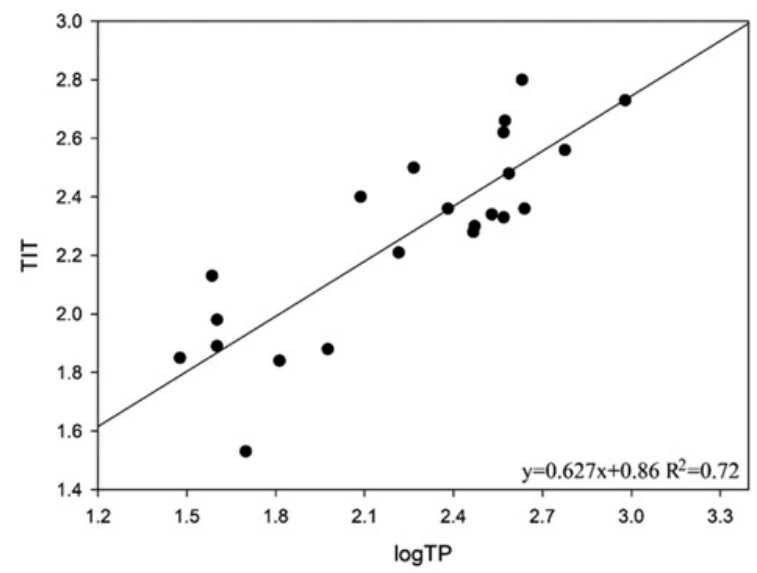

(a)

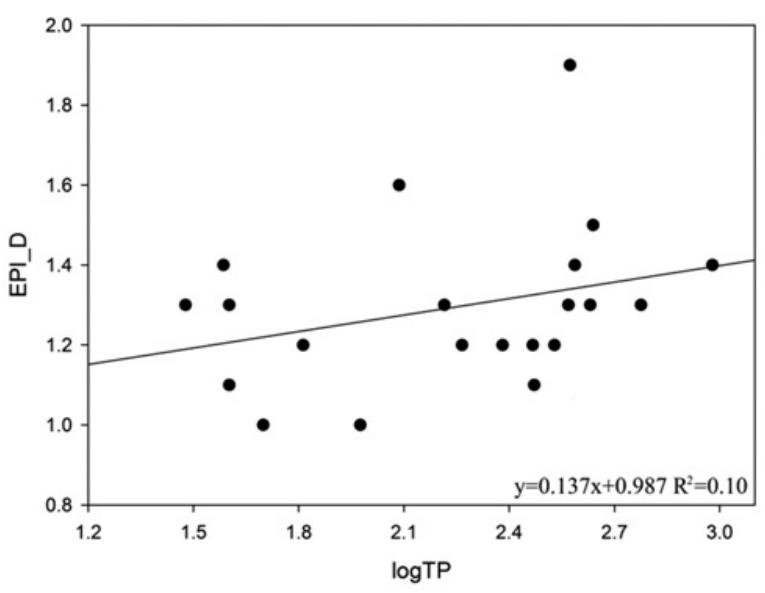

(b)

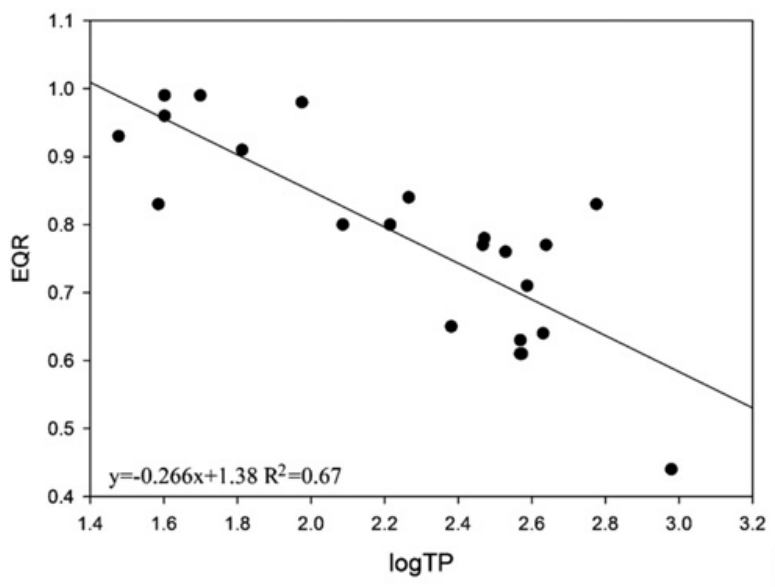

(c)

Fig. 3. Relationships between $\log \mathrm{TP}$ and (a) TIT, (b) EPI-D, and (c) EQR at the sampling stations.

2012; O'Driscoll et al., 2012). The number of species was higher than that in certain previous studies (e.g., Bellinger et al., 2006; O'Driscoll et al., 2012).

CCA was effective in explaining diatom species-environment relationships. The results of CCA showed that Homoeocladia umbonata (Ehrenberg) Kuntze, Navicula cryptocefalsa Lange-Bertalo, and Stephanocyclus meneghinianus (Kützing) Skabitschevsky were associated with high nutrient concentrations (Fig. 2). H. umbonata (Ehrenberg)
Kuntze has been indicated as a species of shallow enriched turbid waters including rivers (Reynolds et al., 2002; Padisák et al., 2009). N. cryptocefalsa Lange-Bertalo has been indicated to be a nutrient-tolerant species (Ács et al., 2004) and tolerant to organic pollution and eutrophication (Salomoni et al., 2006). S. meneghinianus (Kützing) Skabitschevsky has been considered as a pollutiontolerant species (Venkatachalapathy and Karthikeyan, 2013) and as a species typical of polluted environments (Sladeček, 1973; Kobayasi and Mayama, 1989; Van Dam et al., 1994; Lobo et al., 1996, 2002; Salomoni et al., 2006). On the other hand, the CCA indicated that $F$. capucina Desmazières, C. excisa Kützing, and Schizonema radiosum (Kützing) Kuntze preferred low nitrogen content, especially $\mathrm{TN}, \mathrm{N}-\mathrm{NO}_{3}$, and $\mathrm{N}-\mathrm{NO}_{2}$. F . capucina has been indicated as a pollution-sensitive species (Bere and Tundisi, 2011) and characteristic of good status (Kelly et al., 2008). C. excisa Kützing has been considered to be dominant in I-II water quality (less polluted) (Gómez and Licursi, 2001). F. capucina Desmazières and C. excisa Kützing are high profile species that dislike high TSS (Marcel et al., 2017) and are good competitors for nutrients (Rimet et al., 2016). S. radiosum (Kützing) Kuntze has been reported as a sensitivity indicator species (Wang et al., 2014). The occurrence of the species mentioned (pollution-tolerant or -sensitive) can reflect the integrated effect of different pressures and the water quality of the water bodies reflected by organic and inorganic pollutants.

\section{Test of the TIT and comparison with EPI-D}

The present study is the first attempt to assess the ecological status in running waters in western Anatolia using a new diatom index (TIT) according to the WFD. Relationships between TIT and EPI-D and $\log$ TP (Fig. 3(a) and (b)) indicated that TIT was better correlated to $\operatorname{logTP}\left(R^{2}=0.72, P<0.01\right)$ than EPI-D $\left(R^{2}=0.10\right)$. Moreover, the EQR values based on TIT also showed a significant negative correlation with $\log \mathrm{TP}\left(R^{2}=0.67\right.$, $P<0.01)$. In relation to the ecological status based on the TIT, a poor status was found in Dalaman stream (A8) which had the highest nutrient contents (TN, TP; $-\mathrm{NO}_{3}$, $\mathrm{N}-\mathrm{NO}_{2}$, and $\mathrm{P}-\mathrm{PO}_{4}$ ). The ecological status of this station was confirmed by the presence of pollution-tolerant or eutrophication indicator species such as $H$. umbonata (Ehrenberg) Kuntze, N. cryptocefalsa Lange-Bertalo and $S$. meneghinianus (Kützing) Skabitschevsky. On the other hand, a high ecological status was observed in Kaya, Kocabük, Karabeyyurdu, R1, and Delin creeks. These ecosystems also had low nutrient concentrations and undisturbed hydromorphological characteristics, representing reference condition water body types as required by the WFD (Directive, 2000). High ecological status has also been reported in the lowland streams of Northern Italy (Beltrami et al., 2012) using other diatom indices (IPS; CEMAGREF, 1982 and TI; Rott et al., 1999) and some rivers in Ireland by O'Driscoll et al. (2012) using the 
revised TDI (Kelly et al., 2008). In fact, these water bodies as well as our studied sites were located at similar altitudes $(<400 \mathrm{~m})$. This situation and the occurrence of the same cosmopolitan diatom taxa observed in both our sites and above-mentioned watercourses could explain the similarity in the ecological status of the sites.

\section{Conclusion}

A total of 102 diatoms species from 25 running water bodies were identified during the present study. F. capucina Desmazières, C. exisa Kützing, Gomphonema parvulum Kützing, U. ulna (Nitzsch) Compère, and C. placentula Ehrenberg were commonly found in the sampling stations. The effects of multiple complex environmental variables on the diatom assmenblages were elucidated using multivariate analyses. The ecological statuses obtained for the different sites based on the trophic index correspond exactly to the state of deterioration of these water bodies due to human activities such as discharge of waste water, agriculture, and fisheries. The results of this study provide a contribution to the basin approach, and the TIT as a biological metric could be a useful tool for the assessment of running waters in Mediterranean countries.

Acknowledgements. This study was supported by the Directorate General for Water Management of the Ministry of Forestry and Water Affairs (Republic of Turkey) (Project no: 20011K050400), DOKAY-ÇED Company, and the Scientific Research Projects Executive Council of the University of Gaziantep.

\section{References}

Ács É., Szabó K., Tóth B. and Kiss K.T., 2004. Investigation of benthic algal communities, especially diatoms of some Hungarian streams in connection with reference conditions of the Water Framework Directives. Acta Bot. Hung., 46, 255-277.

APHA, 2012. American Public Health Association. American Water Works Association, Water Environment Federation, Standard Methods for the Examination of Water and Wastewater (22nd edn,), 1496 p.

Bellinger B.J., Christine C. and Catherine M.O., 2006. Benthic diatoms as indicators of eutrophication in tropical streams. Hydrobiologia, 573, 75-87.

Beltrami M.E., Ciutti F., Cappelletti C., Losch B., Alber R. and Ector L., 2012. Diatoms from Alto Adige/Sudtirol (Northern Italy): characterization of assemblages and their application for biological quality assessment in the context of the Water Framework Directive. Hydrobiologia, 695, 153-170.

Bere T. and Tundisi J.G., 2011. Diatom-based water quality assessment in streams influence by urban pollution: effects of natural and Two selected artificial substrates, São Carlos-SP, Brazil. Braz. J. Aquat. Sci. Technol., 15(1), 54-63.

Billen G., Garnier J., Ficht A. and Cun C., 2001. Modelling response of water quality in the Seine river estuary to human activity in its watershed over the last 50 years. Estuaries, 24, 977-993.

Bona F., Falasco E., Fassina S., Griselli B. and Badino G., 2007. Characterization of diatom assemblages in mid-altitude streams of NW Italy. Hydrobiologia, 583, 265-274.

Çelekli A., Toudjani A.A., Kayhan S., Lekesiz H.Ö. and Gümüş E.Y., 2017. Ülkemize Özgü Su Kalitesi Ekolojik Değerlendirme Sisteminin Kurulmasi Projesi (project no: 20011K050400). T.C. Orman ve Su İşleri Bakanliği Su Yönetimi Genel Müdürlüğü.

CEMAGREF, 1982. Etude de Méthodes Biologiques Quantitatives d'Appréciation de la Qualité des Eaux. Rapport Q.E.Lyon- A.F.B.Rhône-Mediterrannee-Corse.

Delgado C. and Pardo I., 2014. Comparison of benthic diatoms from Mediterranean and AtlanticSpanish streams: community changes in relation to environmentalfactors. Aquat. Bot., 120, 304-314.

Della Bella V., Pace G., Barile M., Zedde A., Puccinelli C., Ciadamidaro S., Danieli P.P., Andreani P., Aulicino F.A., Belfiore C. and Mancini L., 2012. Benthic diatom assemblages and their response to human stress in small-sized volcanic-siliceous streams of central Italy (Mediterranean eco-region). Hydrobiologia, 695, 207-222.

Dell'uomo A., 1999. Use of algae for monitoring rivers in Italy: current situation and perspectives. In: Prygiel J., Whitton B.A. and Bukowska J. (eds.), Use of algae for monitoring rivers III, Agence de l'Eau Artois-Picardie, Douai, 17-25.

Dell'uomo A., 2004. L'indice diatomico di eutrofizzazione/ polluzione (EPI-D) nel monitoraggio delle azque correnti, line guida. Dipartimento di Botanica ed Ecologia, Universita di Camerino.

Directive, 2000. Directive 2000/60/EC of the European parliament and of the council of 23 October 2000 establishing a framework for community action in the field of water policy. O.J.E.C., 327, 1-72.

Ducharne A., Baubion C., Beaudoin N., Benoit M., Billen G., Brisson N., Garnier J., Kieken H., Lebonvallet S., Ledoux E., Mary B., Mignolet C., Poux X., Sauboua E., Schott C., Théry S. and Viennot P., 2007. Long term prospective of the Seine River system: confronting climatic and direct anthropogenic changes. Sci. Total Environ., 375, 292-311.

European Committee for Standardization, 2003. Water quality Guidance standard for the routine sampling and pretreatment of benthic diatoms from rivers. European Standard EN, 14407, 14 p. Brussels.

European Committee for Standardization, 2004a. Water quality - Guidance standard for the identification, enumeration and interpretation of benthic diatoms from running waters. European Standard EN, 13946, 12 p. Brussels.

European Committee for Standardization, 2004b. Water quality - Guidance standard for the surveying, sampling and laboratory analyses of phytobenthos in shallow running water. European Standard EN, 15708, 22 p. Brussels.

European Communities (EC), 2009. Water Framework Directive intercalibration technical report. Part 2. In: Poikane S. (ed.), Lakes. European Commission, Ispra, Italy. Joint Research Centre.

Gómez N. and Licursi M., 2001. The Pampean Diatome Index (PDI) for assessment of rivers and streams in Argentina. Aquat. Ecol., 35, 173-181. 
Hering D., Johnson R.K., Kramm S., Schmutz S., Szoszkiewicz K. and Verdonschot P.F.M., 2006. Assessment of European streams with diatoms. Macrophytes, macroinvertebrates and fish: a comparative metric-based analysis of organismresponse to stress. Freshw. Biol., 51, 1757-1785.

Hlúbiková D., Hindáková A., Matúš H. and Miettinen J., 2007. Application of diatom water quality indices in influenced and non-influenced sites of Slovak rivers (Central Europe). Arch. Hydrobiol. Suppl., 161(3-4), 444-460.

John D.M., Whitton B.A. and Brook J.A., 2002. The Freshwater Algal Flora of the British Isles (1st edn), Cambridge University Press, Cambridge.

Kelly M., Juggins S., Guthrie R., Pritchard S., Jamieson J., Rippey B., Hirst H. and Yallop M., 2008. Assessment of ecological status in U.K. rivers using diatoms. Freshw. Biol., 53, 403-422.

Kelly M.G. and Whitton B.A., 1995. The trophic diatom index: a new diatom index for monitoring eutrophication in rivers. J. Appl. Phycol., 7, 433-444.

Kelly M.G., Cazaubon A., Coring E., Dell'Uomo A., Ector L., Goldsmith B., Guasch H., Laugaste R., Hürlimann J., Jarlman A., Kawecka B., Kwandrans J., Lindstrøm E.A., Leitao M., Marvan P., Padisák J., Pipp E., Prygiel J., Rott E., Sabater S., VanDam H. and Vizinet J., 1998. Recommendations for the routine sampling of diatoms for water quality assessments in Europe. J. Appl. Phycol., 10(2), 215-224.

Kobayasi H. and Mayama S., 1989. Evaluation of riverwater quality by diatoms. Korean J. Phycol., 4, 121-133.

Krammer K, 2000. The Genus Pinnularia. In: Lange-Bertalot H. (ed.), Diatoms of Europe-Diatoms of the European Inland Waters and Comparable Habitats. Gantner Verlag, Ruggel, $1,1-703$.

Krammer K., 2002. The Genus Cymbella. In: Lange-Bertalot H. (ed.), Diatoms of Europe - Diatoms of the European Inland Waters and Comparable Habitats. Gantner Verlag, Ruggel, $3,1-584$.

Krammer K. and Lange-Bertalot H., 1991a. Bacillariophyceae. 3 Teil: Centrales. Fragilariaceae. Eunotiaceae. In: Ettl H., Gerloff J., Heynig H. and Mollenhauer D. (eds.), Süßwasser-flora von Mitteleuropa. Band 2. Fischer Verlag, Stuttgart.

Krammer K. and Lange-Bertalot H., 1991b. Bacillariophyceae. 4 Teil: Achnanthaceae. Kritische Erganzungen zu Navicula (Lineolatae) und Gomphonema. In: Ettl H., Gartner G., Gerloff J., Heynig H. and Mollenhauer D. (eds.), Süßwasserflora von Mitteleuropa. Band 2. Fischer Verlag, Stuttgart.

Krammer K. and Lange-Bertalot H., 1999a. Bacillariophyceae. 1 Teil: Naviculaceae. In: Ettl H., Gerloff J., Heynig H. and Mollenhauer D. (eds.), Süßwasserflora von Mitteleuropa. Band 2. Akademischer Verlag, Heidelberg, Berlin.

Krammer K. and Lange-Bertalot H., 1999b. Bacillariophyceae. 2 Teil: Bacillariaceae. Epithemiaceae. Surirellaceae. In: Ettl H., Gerloff J., Heynig H. and Mollenhauer D. (eds.), Süß-wasserflora von Mitteleuropa. Band 2. Akademischer Verlag, Heidelberg, Berlin.

Lange-Bertalot H., 2001. Navicula sensu stricto 10 Genera Separated from Navicula sensu lato Frustulia. In: LangeBertalot H. (ed.), Diatoms of Europe -Diatoms of the European Inland Waters and Comparable Habitats. Gantner Verlag, Ruggel, 2, 1-526.
Leelahakriengkrai P. and Peerapornpisal Y., 2014. Evaluation of the Trophic Benthic Diatom Index in some Main Rivers of Thailand. Adv. Environ. Biol., 8(1), 248-254.

Leira M., Chen G., Dalton C., Irvine K. and Taylor D., 2009. Patterns in freshwater diatom taxonomic distinctness along an eutrophication gradient. Freshw. Biol., 54, 1-4.

Leps J. and Smilauer P., 2003. Multivariate Analysis of Ecological Data using CANOCO. Cambridge University Press, Cambridge.

Lobo E.A., Callegaro V.L.M., Oliveira M.A., Salomoni S.E., Schuler S. and Asai K., 1996. Pollution Tolerant Diatoms from lotic Systems in the Jacuí Basin, Rio Grande do Sul, Brasil. Iheringia. Sér. Bot. Porto Alegre, 47, 45-72.

Lobo E.A., Callegaro V.L.M. and Bender P., 2002. Utilizaça? de algas diatomáceas epilíticas como indicadoras da qualidade da água em rios e arroios da Regiaõ Hidrográfica do Guaíba, RS, Brasil. EDUNISC, Santa Cruz do Sul, 127 p.

Mancini L. and Arcá G., 2000. Carta della Qualità Biologica dei Corsi d'Acqua della Regione Lazio. Regione Lazio. Assessorato Ambiente. Dipartimento Ambiente e Protezione Civile, $156 \mathrm{p}$.

Marcel R., Berthon V., Castets V., Rimet F., Thiers A., Labat F. and Fontan B., 2017. Modelling diatom life forms and ecological guilds for river biomonitoring. Knowl. Manag. Aquat. Ecosyst., 418, 1.

Mustapha M.K., 2008. Assessment of the Water Quality of Oyun Reservoir., Offa., Nigeria. Using selected physico-chemical parameters. Turk. J. Fish. Aquat. Sci., 8, 309-319.

O’Driscoll C., de Eyto E., Rodgers M., O'Connor M., Asam Z.Z. and Xiao L., 2012. Diatom assemblages and their associated environmental factors in upland peat forest rivers. Ecol. Indic., 18, 443-451.

Padisák J., Borics G., Grigorszky I. and Soróczki-Pintér E., 2006. Use of phytoplankton assemblages for monitoring ecological status of lakes within the Water Framework Directive: the assemblage index. Hydrobiologia, 553, 1-14.

Padisák J., Crossetti L. and Naselli-Flores L., 2009. Use and misuse in the application of the phytoplankton functional classification: a critical review with updates. Hydrobiologia, 621, 1-19.

Pan Y. and Stevenson R.J., 1996. Gradient analysis of diatom assemblages in western Kentucky wetlands. J. Phycol., 32, 222-232.

Potapova M. and Charles D.F., 2003. Distrivution of benthic diatoms in US rivers in relation to conductivity and ionic composition. Fresw. Biol., 48, 1311-1328.

Potapova M., Charles D.F., Ponade K.C. and Winter D.M., 2004. Quantifying speciesindicator values for trophic diatom indices: comparison of approaches. Hydrobiologia, 517, 25-41.

Reynolds C.S., Huszar V., Kruk C., Naselli-Flores L. and Melo S., 2002. Towards a functional classification of the freshwater phytoplankton, review. J. Plankton Res., 24(5), $417-428$.

Rimet F., 2012. Recent views on river pollution and diatoms. Hydrobiologia, 683, 1-24.

Rimet F., Bouchez A. and Tapolczai K., 2016. Spatial heterogeneity of littoral benthic diatoms in a large lake: monitoring implications. Hydrobiologia, 771, 179-193.

Rott E., Pipp E., Pfister P., Van Dam H., Ortler K.,Binder N. and Pall K., 1999. Indikationslisten für Aufwuchsalgen in 
österreichischen Fliessgewässern. Teil 2: Trophieindikation (sowie geochemische Präferenzen, taxonomische und toxikologische Anmerkungen). Wasserwirtschaftskataster, Bundesministerium f.Land-u. Forstwirtschaft, Wien, 248 p.

Rott E., Pipp E. and Pfister P., 2003. Diatom methods developed for river quality assessment in Austria and a cross-chech against numerical trophic indication methods used in Europe. Algol. Stud., 110, 91-115.

Round F.E., 1991. Diatoms in river water-monitoring studies. $J$. Appl. Phycol., 3, 129-145.

Salomoni S.E., Rocha O., Callegaro V.L. and Lobo E.A., 2006. Epilithic diatoms as indicators of water quality in the Gravataí river, Rio Grande do Sul, Brazil. Hydrobiologia, 559, 233-246.

Schindler D.W., 2006. Recent advances in the understanding and management of eutrophication. Limnol. Oceanogr., 51(1), $356-363$.

Sladeček V., 1973. System of water quality from the biological point of view. Archiv für Hydrobiologie Beiheft, 7, $1-218$.

Smol J.P., 2008. Pollution of Lakes and Rivers: A Paleoenvironmental Perspective. Blackwell Publishing, Oxford.

ter Braak C.J.F. and Šmilauer P., 2002. CANOCO Reference Manual and CanoDraw for Windows User's Guide:
Software for Canonical Community Ordination (version 4.5). Microcomputer Power Press, Ithaca.

Vadeboncoeur Y. and Steinman A.D., 2002. Periphyton function in lake ecosystems. Sci. World J., 2, 1449-1468.

Van Dam H., Mertens A. and Sinkeldam J., 1994. A coded checklist and ecological indicator values of freshwater diatoms from The Netherlands. Neth. J. Aquat. Ecol., 28, $117-184$

Venkatachalapathy R. and Karthikeyan P., 2013. Benthic Diatoms in River Influenced By Urban Pollution, Bhavani Region, Cauvery River, South India. I.J.I.T.E.E., 2(3), 206-210.

Wallis C., Seon-Massin N., Martini F. and Schouppe M., 2011. Implementation of the Water Framework Directive: When Ecosystem Services Come into Play. Brussels.

Wang X., Zheng B., Liu L. and Li L., 2014. Use of Diatoms in River Health Assessment. Annu. Res. Rev. Biol., 4(24), 4054-4074.

Wunsan S., Cattaneo A. and Bourassa N., 2002. Comparing diatom species. genera andsize in biomonitoring: a case study form streams in the Laurentians (Québec.Canada). Freshw. Biol., 47, 325-340.

Zelinka M. and Marvan P., 1961. Zur Präzisierung der biologischen Klassifikation der Reinheit fliessender Gewässer. Archiv. Hydrobiol., 57, 389-407. 